\title{
Towards a Less Destructive Crossover Operator Using Immunity Theory
}

\author{
Yingzhou Bi ${ }^{1,2}$, Lixin Ding ${ }^{1}$, and Weiqin Ying ${ }^{1}$ \\ ${ }^{1}$ State key laboratory of software engineering, Wuhan University, Wuhan 430072, China \\ ${ }^{2}$ Department of Information Technology, Guangxi Teachers Education University, \\ Nanning 530001, China \\ byzhou@163.com
}

\begin{abstract}
When searching for good scheme, a good solution can be destroyed by an inappropriate choice of crossover points. Furthermore, because of the randomicity of crossover, mutation and selection, a better solution can hardly reach in last stage in EA, and the solution always traps in local optimal. Faced to "exploding" solution space, it is tough to find high quality solution just by increasing the population size, diversity of searching, and the number of iteration. In this paper, we design the immunity operator to improve the crossover result by utilizing the immunity theory. As the "guided mutation operator", the immunity operator substituted the "blind mutation operator" in normal EA, to restrain the degenerate phenomenon during the evolutionary process. We examine the algorithm with examples of TSP and gain promising result.
\end{abstract}

Keywords: Crossover operator, Immunity operator, Traveling salesman problem.

\section{Introduction}

The role of crossover in evolutionary algorithms is to create new individuals from old ones [1]. During the process of stochastic search, crossover is considered to be the major driving force behind EA. However, when searching for good scheme, standard crossover is widely accepted as being a largely destructive operator[2]; a simple one point crossover operator generates an individual by selecting a crossover point randomly from a parent, then splitting both parents at this point and creating two children by exchanging the tails. The randomness of this operator makes it mostly destructive (generates children inferior to their parents).

Classic EA uses little problem specific knowledge, so its' efficiency is usually low. In order to improve EA's efficiency, we try to have other methods or data structures incorporated into it. This category of algorithm is very successful in practice and forms a rapid growing research area with great potential [3-4]. In order to improve the algorithmic efficiency, the immune genetic algorithm (IGA) is presented in [5], which utilizes some characteristics and knowledge in the pending problems for restraining the degenerative phenomena during evolution.

In this paper, we firstly analyze the destructive and constructive aspects of crossover and mutation in section 2, and then design a novel immunity genetic algorithm in section 3: the "blind mutation operator" is substituted by the "guided mutation operator". 
Finally, we compare the novel algorithm and canonical genetic algorithm with the example of traveling salesman problem.

\section{Disruption and Construction of Crossover}

The disruption of crossover refers to the offspring of an individual in a schema $H_{\mathrm{k}}$ no longer belong to schema $H_{\mathrm{k}}$ after crossover. It can be depicted as fig.1: $P_{1}$ is a member of a third-order schema $H_{3}$, and $P_{2}$ is an arbitrary string. If the two parents have not matching alleles at position $d_{2}$ or they have not matching alleles at poison $d_{1}$ and $d_{3}$, the schema $H_{3}$ should be disrupted.

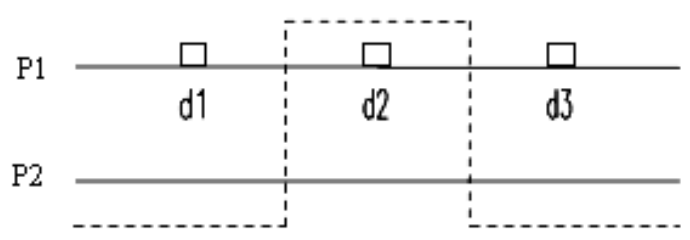

Fig. 1. An example of two-point crossover. $P_{1}$ is a member of a third-order schema $H_{3}$, and $P_{2}$ is an arbitrary string.

The construction of crossover refers to having crossover create an instance of a schema $H_{\mathrm{k}}$ from both parents, where one parent is in schema $H_{\mathrm{m}}$, while the other parent is the schema $H_{\mathrm{n}}$, and $k=m+n$. Figure 2 provides a pictorial example. $P_{1}$ is a member of a second-order schema, and $P_{2}$ is a member of another second-order schema. A higher schema $H_{4}$ is constructed by crossover.

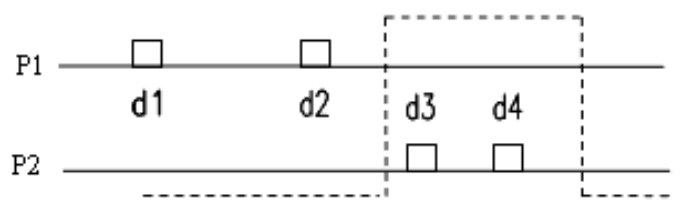

Fig. 2. An example of construction of two-point crossover. $P_{1}$ is a member of a two-order schema $H_{2}$, and $P_{2}$ is a member of another two-order schema $H_{2}$.

Based on the systemic analyze of crossover and mutation, literature [6] presents some general results: (1) all forms of crossover are more disruptive of high-order schemas and become less disruptive when the population converges.(2) more disruptive crossover are more likely to construct(and vice versa). This has proven a NoFree-Lunch Theorem with respect to the disruption and construction aspects of crossover (3) Mutation can achieve any level of disruption that crossover can achieve, however, from a construction point of view; crossover is more powerful than mutation. 


\section{A Novel Algorithm with Immunity Operator as the "Guided Mutation Operator"}

The most remarkable role of immune system is the protection of the organism against the attack of disease, and the elimination of malfunctioning cells. Inspired by the theory of immunity, one main idea of immunity computation is to abstract some useful problem-specific knowledge (vaccine) from the pending problem (antigen), and utilize this knowledge to search the solution (antibody). In TSP, a solution with high fitness should be composed of most short links between nodes, it means the usher and subsequence of a node in a roundtrip is always its near neighbor, and here, we called it "principle of near neighbor". If the principle is disobeyed, the roundtrip will be composed of lots of long links, and the total length must be very long. So we should find the near neighbor of all nodes at the beginning of algorithm and the near neighbor of nodes are selected by the distance between them. Usually, we only consider five nodes as near neighbor. The principle of near neighbor and the near neighbor of every node are the vaccine for TSP.

\subsection{Vaccinations}

Vaccination means modifying the genes on some bits of the candidate solution with priori knowledge.

1) Analyze all links in the candidate roundtrip: record each pair of nodes of the long links (if a solution composing $n$ links, we usually consider $n / 2 \sim n / 3$ longer links) and the distance between them with a structure array. Here we called the structure array update-array; every element in update-array $A$ has three parts: two nodes and a distance, where one node is called resource-nodes and another node is called destinynodes. The update-array $A$ is sorted in descend-order according the distance in its element.

2) Examine all the resource-node in update-array $A$ whether the destiny-node is its near neighbor. For example, for any element in update-array $A$, suppose its resourcenode is $V_{\mathrm{a}}$, and its destiny-node is $V_{\mathrm{b}}$, if $V_{\mathrm{b}}$ is not near neighbor of $V_{\mathrm{a}}$, it means something is wrong in this gene, repairing this gene may increase the fitness of the candidate solution. Inspired by 2-change and 3-change algorithms, which have a time complexity of $O\left(n^{2}\right)$ and $O\left(n^{3}\right)$ respectively [7], we present 2-repair and 3-repair operators to repair the malfunction genes in candidate solution, however their time complexity are $O(n)$,for every node we only consider its near neighbor and the number of near neighbor is usually 5 8.

3) 2-repair operator: for a long link $\left(V_{\mathrm{a}}, V_{\mathrm{b}}\right)$,if the destiny-node $V_{\mathrm{b}}$ is not 2-nearest neighbor of resource-node $V_{\mathrm{a}}$, then select a node $V_{\mathrm{c}}$ from the near neighbor of $V_{\mathrm{a}}$, obviously we have $d\left(V_{\mathrm{a}}, V_{\mathrm{b}}\right)>d\left(V_{\mathrm{a}}, V_{\mathrm{c}}\right)$. Suppose node $V_{\mathrm{d}}$ is subsequence of node $V_{\mathrm{c}}$ and the solution is expressed as $\pi=\left\{V_{1}, V_{2}, \ldots V_{\mathrm{a}}, V_{\mathrm{b}} \ldots V_{\mathrm{c}}, V_{\mathrm{d}} \ldots V_{\mathrm{n}}\right\}$, its total length is

$$
D(\pi)=\sum_{i=1}^{r} d_{i}+d\left(V_{a}, V_{b}\right)+\sum_{i=r+2}^{t} d_{i}+d\left(V_{c}, V_{d}\right)+\sum_{i=t+2}^{n} d_{i} .
$$


if $d\left(V_{\mathrm{a}}, V_{\mathrm{b}}\right)+d\left(V_{\mathrm{c}}, V_{\mathrm{d}}\right)>d\left(V_{\mathrm{a}}, V_{\mathrm{c}}\right)+d\left(V_{\mathrm{b}}, V_{\mathrm{d}}\right)$, then we reverse all the nodes between $V_{\mathrm{b}}$ and $V_{\mathrm{c}}\left(\right.$ including $\left.V_{\mathrm{b}}, V_{\mathrm{c}}\right)$, then get a new solution $\pi^{\prime}=\left\{V_{1}, V_{2}, \ldots V_{\mathrm{a}}, V_{\mathrm{c}} \ldots V_{\mathrm{b}}, V_{\mathrm{d}} \ldots V_{\mathrm{n}}\right\}$, its total length is

$$
D\left(\pi^{\prime}\right)=\sum_{i=1}^{r} d_{i}+d\left(V_{a}, V_{c}\right)+\sum_{i=r+2}^{t} d_{i}^{\prime}+d\left(V_{b}, V_{d}\right)+\sum_{i=t+2}^{n} d_{i}
$$

for the symmetry TSP, we always have $d_{i}=d_{i}$ ' , so under the condition $d\left(V_{\mathrm{a}}, V_{\mathrm{b}}\right)+$ $d\left(V_{\mathrm{c}}, V_{\mathrm{d}}\right)>d\left(V_{\mathrm{a}}, V_{\mathrm{c}}\right)+d\left(V_{\mathrm{b}}, V_{\mathrm{d}}\right)$, it is obvious that $\mathrm{D}(\pi)>\mathrm{D}\left(\pi^{\prime}\right)$, which means the new solution is better.
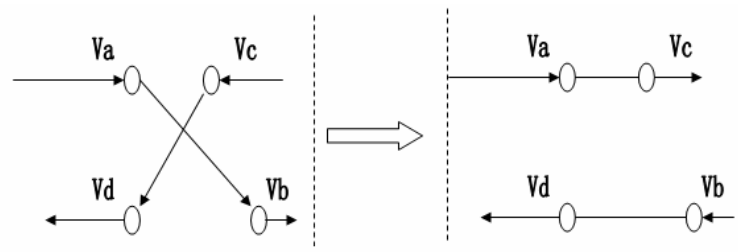

Fig. 3. An example of vaccination of 2-repair for TSP

4) 3-repair operator: it is similar to 2-repair, but it exchange 3 links in every repairing. The mechanism is pictorial in figure 4: the original solution is $\pi=\left\{V_{1}, V_{2}, \ldots V_{\mathrm{a}}, V_{\mathrm{b}} \ldots V_{\mathrm{c}}, V_{\mathrm{d}}, V_{e} \ldots V_{\mathrm{n}}\right\}$,

if $d\left(V_{\mathrm{a}}, V_{\mathrm{b}}\right)+d\left(V_{\mathrm{c}}, V_{\mathrm{d}}\right)+d\left(V_{\mathrm{d}}, V_{\mathrm{e}}\right)>d\left(V_{\mathrm{a}}, V_{\mathrm{d}}\right)+d\left(V_{\mathrm{d}}, V_{\mathrm{b}}\right)+d\left(V_{\mathrm{c}}, V_{\mathrm{e}}\right)$ then exchange 3 links: the 3 bold links are substituted by 3 dash links, and the new solution become $\pi^{\prime}=\left\{V_{1}, V_{2}, \ldots V_{\mathrm{a}}, V_{\mathrm{d}}, V_{\mathrm{b}} \ldots V_{\mathrm{c}}, V_{e} \ldots V_{\mathrm{n}}\right\}$.It is obvious that $\mathrm{D}(\pi)>\mathrm{D}\left(\pi^{\prime}\right)$.

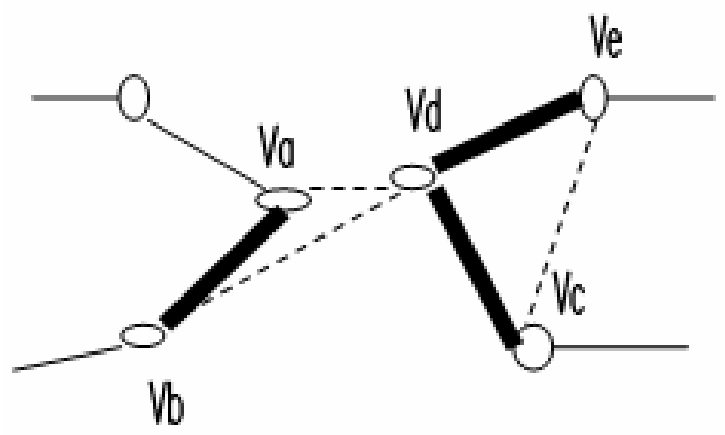

Fig. 4. An example of vaccination of 3-repair for TSP

\subsection{A Novel Algorithm for TSP}

According to the analysis in section 2 , from a construction point of view, crossover is more powerful than mutation. We present a novel algorithm for TSP: the "blind" mutation operator in canonical genetic algorithm is substituted by the "guided" mutation 
operator, which is immunity operator. The aim of leading immune concepts and methods into GA is to utilize the problem-specific knowledge for refraining the destruction of crossover while make useful of its construction during evolutionary process.

\section{Algorithm 1}

1) Initialize the population with greedy algorithm.

2) Abstract the vaccine.

3) Evaluate the individuals, if the solution quality is satisfied then stop, or if reach the maximal iteration time but the solution quality is not satisfied, then repair the best individual with 3-repair operator(in section 3.1) and stop; otherwise, continue the following.

4) Crossover: select two parents and perform crossover.

5) Vaccination: repair the resulting offspring with 2-repair operator (in section 3.1) with probability $P \mathrm{i}$, and insert the new candidates into next generation.

6) Go to step 3 .

\section{Experimental Results}

We examine algorithm 1 and canonical genetic algorithm(CGA in brief, it's crossover operator is the Order Crossover designed by Davis, and mutation operator is the Inversion Mutation [1].) with 4 TSP instances: two of them are TSP200 and TSP500, where the nodes in TSP200 and TSP500 are 200 and 500 respectively; The location of all the nodes are generated randomly in two-dimension space: $x \in[0,1000], y \in[0,1000]$, the distance between nodes is Euclidean distance. Another two instances are fl417, att532, which come from TSPLIB [8]. The experiments are carried on a PC with PIV 2.93 GHz CPU, 1G RAM and Windows XP operation system.

In order to compare the performance between algorithm 1 and CGA, we design the parameter as following: in CGA, the size of population is 100; the crossover and the mutation probabilities are 0.5 and 0.4 ,respectively; the number of generations is 20000; in algorithm 1, the size of population is 50; the crossover and the immunity probabilities are 0.8 and 0.85 ,respectively;the number of generations is 100 .The experimental results of CGA and algorithm 1 over 20 independent runs respectively are listed in Table 1, where $n$ is the number of nodes, $D_{0}$ is the known minimal tour length: the results are gained by Concorde package [9] (in att532 the minimal tour length by Concorde package is 86729 while literature [10] take it as 87550 )and time denotes the average run time, while $\delta$ denotes the relative difference[10] which is defined in Equation (3)

$$
\delta \%=\left(\sum_{i=1}^{T}\left(D_{i}-D_{0}\right)\right) /\left(T \times D_{0}\right) \times 100 \%
$$

Where $D_{\mathrm{i}}$ denotes the minimal tour length obtained from the $i$-th run, $T$ is the number of independent runs. 
Table 1. Compare the performance between CGA and algorithm 1

\begin{tabular}{l|c|c|c|c|c|c}
\hline TSP & \multirow{2}{*}{$n$} & \multirow{2}{*}{$D_{0}$} & \multicolumn{2}{|c|}{$\delta$} & \multicolumn{2}{|c}{ Time(second) } \\
\cline { 4 - 7 } Instances & & & CGA & Algorithm1 & CGA & Algorithm 1 \\
\hline TSP200 & 200 & 10610 & 7.9 & $\mathbf{0 . 3}$ & 136 & $\mathbf{1 0}$ \\
TSP500 & 500 & 16310 & 4.4 & $\mathbf{1 . 1}$ & 425 & $\mathbf{9 9}$ \\
Fl417 & 417 & 11861 & 2.9 & $\mathbf{1 . 2}$ & 315 & $\mathbf{3 9}$ \\
Att532 & 532 & 86729 & 6.4 & $\mathbf{1 . 7}$ & 473 & $\mathbf{1 0 8}$ \\
\hline
\end{tabular}

For the analysis of the role of crossover and immunity operator in immunityevolutionary algorithm, we perform the algorithm 1 with different crossover probabilities $P_{\mathrm{c}}$ while the immunity probability $P_{\mathrm{i}}$ is always 0.85 , given population size $=50$, iterative times $=100$. The experimental results of algorithm 1 over 20 independent runs respectively are shown in table 2 , where $\delta$ denotes the relative difference [10] which is defined in Equation (3).

Table 2. Compare the role of crossover and immunity operator in algorithm 1

\begin{tabular}{l|r|c|c|c}
\hline \multirow{2}{*}{$\begin{array}{l}\text { TSP } \\
\text { Instances }\end{array}$} & \multicolumn{4}{|c}{$\delta$} \\
\cline { 2 - 5 }$P_{\mathrm{c}}=0.00$ & $P_{\mathrm{c}}=0.50$ & $P_{\mathrm{c}}=0.80$ & $P_{\mathrm{c}}=0.99$ \\
\hline TSP200 & 7.1 & 1.0 & 0.3 & 0.3 \\
TSP500 & 3.1 & 1.7 & 1.1 & 1.1 \\
F1417 & 6.1 & 2.1 & 1.2 & 1.1 \\
Att532 & 3.9 & 2.4 & 1.7 & 1.5 \\
\hline
\end{tabular}

Based on the results in table 1 to table 2, we can make some general observations:

Firstly, evolutionary algorithm can reach a relatively good solution from a bad solution after a stochastic trial-and-error. Because of the destruction of crossover and mutation, a better solution quality can hardly reach in last stage in EA, and the solution always trap in local optimal. Faced to "exploding" solution space, it is tough to find high quality solution just by increasing the population size (in CGA the population size is 100 , while it is only 50 in algorithm 1), diversity of searching, and the number of iteration (the maximum number of generations in CGA is 20000, while it is only 100 in algorithm 1). However we can restrain the destruction of crossover by repairing the malfunction genes in candidate solution, and utilize its construction in our novel algorithm with immunity operator as "guided" mutation.

Given different crossover probabilities, algorithm 1 has different performance: the bigger is crossover probability, the better is performance, however, when the crossover probability reaches 0.8 , the performance will increase little, while it need more time. If the crossover probability is 0 , it means the crossover is turned off, the performance is the worst. The experimental results validate the construction of crossover. Furthermore, when compared with the pure artificial immunity algorithm [10] in TSP instance att532, the result of algorithm 1 is better: if consider the minimal tour length is 87550 , the relative difference is $0.7 \%$, while the relative difference is $2.21 \%$ in reference [10]. 


\section{Conclusions and Future Work}

By utilizing the problem-specific knowledge, immunity operator can restrict the degenerative phenomena arising from the evolutionary process, and guaranteeing that the search will concentrate in the regions containing high quality solutions. Artificial immunity theory can contribute with the already established methodologies in order to mutually improve their performances and application domains. It shows that the combination of an evolutionary and a heuristic method--a hybrid evolutionary algorithm-performs better than either of its "parent" algorithms alone.

For future work, we will use the proposed method on other combinational problems or even real optimization problems.

Acknowledgments. This work is supported in part by the National Natural Science Foundation of China (Grant no. 60204001), and Natural Science Foundation of Guangxi (Grant no. 0679018).

\section{References}

1. Eiben, A.E, Smith, J.E.: Introduction to Evolutionary Computing. Berlin Heidelberg New York Press, Springer-Verlag (2003)

2. Majeed, H., Ryan,C.: A Less Destructive, Context-Aware Crossover Operator for GP.Lecture Notes in Computer Science, Vol.3905.Berlin /Heidelberg, Springer-Verlag (2006) 36-48

3. Yao, X., Xu, Y.: Recent Advance in Evolutionary Computation. Journ of Comput Sci \& Technol, 21(1) (2006) 1-18

4. Blum C., Roli A.: Metaheuristics in Combinatorial Optimization: Overview and Conceptual Comparison. ACM Computing Surveys, 35(3)( 2003) 268-308

5. Jiao, L., Wang,L.: A novel genetic algorithm based on immunity. IEEE Transactions on System s, M an, and Cybernetics, 30(5) (2000) 552-561

6. Spears, W.M.: The role of Mutation and Recombination in Evolutionary Algorithms. Virginnia: George Mason University (1998)

7. Helsgaun, K.: An Effective Implementation of the Lin-Kernighan Traveling Salesman Heuristic. http://www.akira.ruc.dk/ keld/

8. http://www.iwr.uni-heidelberg.de/groups/comopt/software/TSPLIB95/

9. Concorde TSP solver for windows. http://www.tsp.gatech.edu/concorde/index.html

10. Gong, M., Jiao, L., Zhang, L.: Solving Tranveling Salesman Problem by Artificial Immunity Responese. T.-D Wang et al.(Eds.):SEAL 2006. Lecture Notes in Computer Science, Vol. 4247. Springer-Verlag, Berlin Heidelberg (2006) 64-71 